\title{
Orthogeriatric Anaesthesia
}

\author{
Stuart M. White
}

\subsection{Introduction}

Traditionally, perioperative care of elderly patients requiring surgical hip fracture fixation was less than exemplary. Patients were administered relatively large amounts of opioid analgesia before surgery, which itself was often delayed for more than $48 \mathrm{~h}$ for 'organisational' or 'anaesthetic' reasons. A significant proportion of patients were not operated on, because the perioperative risk of death was perceived to be too high, and so received conservative management (bedrest). Patients undergoing surgery would be anaesthetised and operated on by junior clinicians, who administered heavy-handed general anaesthesia with opioid analgesia and used a wide variety of surgical techniques and implants. Postoperative care was coordinated by orthopaedic surgeons, and generally delivered in a passive and intermittent manner. Mortality and morbidity were high, and length of postoperative inpatient stay was long.

This approach to care, however, was economically unsustainable given the rapidly changing demographics of developed (and, increasingly, developing) countries. Although the prevalence of hip fracture has remained stable or has fallen slightly, increased longevity has led to an increase in the number of elderly patients presenting with hip fracture. As a result, several European countries began to develop orthogeriatric services, to streamline and coordinate hip fracture care pathways.

This chapter is a component of Part 2: Pillar I.

For an explanation of the grouping of chapters in this book, please see Chapter 1: 'The Multidisciplinary Approach to Fragility Fractures Around the World-An Overview'.

S. M. White $(\bowtie)$

Brighton and Sussex University Hospitals NHS Trust, Brighton, UK 


\subsection{The Relationship Between Anaesthetist and Orthogeriatrician}

Reconfiguration towards multidisciplinary, orthogeriatrician-led care has probably delivered the greatest improvement in hip fracture outcomes in the last two decades. The main benefit of this model is that it allows for continuous, specialised medical care throughout the perioperative period, delivered by anaesthetists and orthogeriatricians.

There are three phases to perioperative care, the preoperative, intraoperative and postoperative phases (Fig. 8.1).

The preoperative phase describes the period from fracture to the patient's arrival in theatres for surgery. Hip fracture is painful, if not always at rest, then usually on movement. Surgical fixation is the only method of providing analgesia and remobilisation in the long term, for which reason it should always be considered an option in preference to non-operative management. Conservative treatment carries the additional risks of immobility - thromboembolism, pressure ulceration and loss of independence. The aim of the preoperative phase, therefore, is to facilitate prompt preparation for surgery. Coordinated orthogeriatric/anaesthetic care enables standardised preoperative assessment (e.g., delivered according to an agreed proforma, detailing history, examination, preoperative investigations and blood crossmatching), risk assessment using scoring systems, analgesia provision according to agreed protocols, fluid resuscitation and organisational and patient-centred preparation for surgery.

Intraoperatively, the aim of anaesthesia is to mitigate the pathophysiological effects of surgery without destabilising the patient's physiology. These patients are at comparatively high risk of perioperative morbidity and mortality, because they

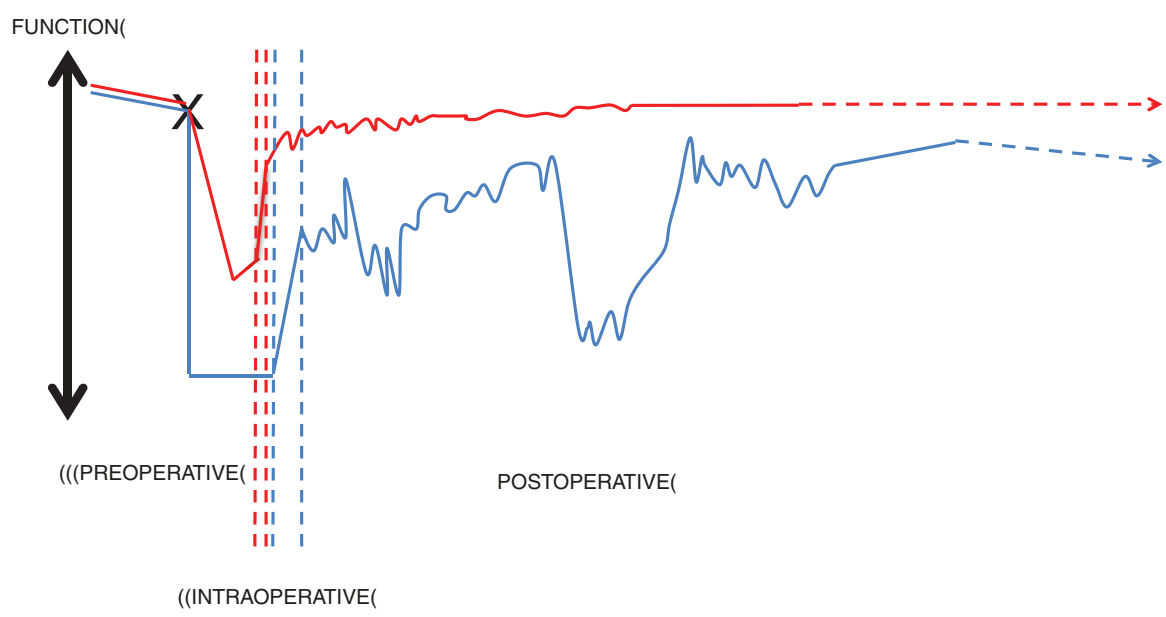

Fig. 8.1 Changes in functional capacity after hip fracture in the three phases of the inpatient episode, with traditional anaesthetic and perioperative care (blue line), compared to proactive multidisciplinary care (red line). See text 
are usually frail and elderly (and have limited physiological reserve), and have one or more comorbidities for which they take one or more drugs; cognitive dysfunction is also common. Conceptually, anaesthesia is less about getting high-risk patients through $0.5-2 \mathrm{~h}$ of major, emergency surgery, but more about normalising the patient's (patho)physiology so that they are able to return to their normal function within hours following the surgery.

National audits have shown that a wide variety of anaesthesia techniques are used because of a result of personal preference and the lack of conclusive evidence for superiority of one technique over another [1, 2]. However, observational studies and meta-analysis indicate certain anaesthesia techniques probably improve the outcome $[3,4]$. Of potentially greater relevance is the idea that hospitals should adopt standardised anaesthesia protocols, so that postoperative care and the management of inevitable complications of anaesthesia and surgery become predictable for orthogeriatricians.

Postoperatively, orthogeriatric care aims to remobilise, re-enable and remotivate patients in preparation for hospital discharge, ideally back to their place of residence before fracture. The early postoperative phase is critical, as delayed remobilisation is associated with a prolonged duration of inpatient stay. Good anaesthesia care facilitates early recovery, by providing non-opioid analgesia, and avoiding delirium, hypotension and anaemia.

Figure 8.1 shows a reconceptualised timeline of what joint anaesthesia/orthogeriatric care should aim to achieve. The blue line represents traditional anaesthesia care. The patient's functional condition has been declining for some time, until they fall and break their hip (' $X$ '), at which point they become entirely dependent. They are taken to hospital but receive minimal care until surgery, and so experience no functional improvement.

Intraoperatively, the fracture is fixed, analgesia, fluids/blood are given, the blood pressure monitored, and the patient's functional status improves, which continues into the immediate postoperative period. However, perhaps the patient develops delirium or feels too nauseous to remobilise for several days in the early postoperative period, as a result of reliance on postoperative opioid analgesia. They recover function in the following few days, but then develop pressure sores or suffer a pulmonary embolism related to their prolonged bedrest, and their functional recovery is delayed again. Eventually, they recover, not quite to their pre-fracture level of function but enough to be discharged from hospital. However, their relatives report that the patient 'was never quite the same' after this episode, with a slow ongoing decline in function after discharge (dotted lines).

In contrast, proactive multidisciplinary care (red line) aims to return patients quickly to their pre-fracture functional status. Simple resuscitation (analgesia, fluids, food) decreases the relative decline in function after fracture, and may indeed begin to improve function preoperatively. The patient undergoes surgery sooner and for a shorter period, during which resuscitation and normalisation of function continues using standardised anaesthesia. The patient's functional status rapidly returns to pre-fracture levels, there are no immobilising complications, the patient is discharged from hospital sooner and remains 'well' after discharge. 


\subsection{Preoperative Care}

International consensus recommendations published by the Fragility Fracture Network in 2018 detail the organisational and interdisciplinary aspects of anaesthesia care that hip fracture patients should be expected to receive in any hospital worldwide [5]. These were endorsed in the 2020 update of the 2012 Association of Anaesthetists UK guidelines, developed in association with the British Geriatrics Society [6]. Recommendations include the delivery of care by a multidisciplinary team of senior clinicians, fast-track hospital admission to an acute orthopaedic/hip ward, the provision of daily, and protected trauma lists that prioritise hip fracture surgery.

Several aspects of preoperative care involve coordinated anaesthetic and orthogeriatric input, including analgesia provision, preoperative preparation and ethical/ legal considerations.

\subsubsection{Preoperative Analgesia}

Hip fractures are usually low impact injuries sustained after a fall from standing height onto osteoporotic bone. Extracapsular fractures (intertrochanteric, subtrochanteric) are more painful than intracapsular fractures (subcapital, transcervical, basicervical), due to the greater degree of periosteal disruption.

Approximately a third of the fractures are associated with mild pain, a third with moderate pain and a third with severe pain. Fractures are usually more painful on movement, for example when the affected leg is raised passively by $20^{\circ}$.

After admission to the hospital, pain is often poorly assessed. Numerical rating scales do not adequately describe pain duration or quality. Assessment needs to take place at rest and on movement, before and after the administration of analgesia. Communication difficulties (deafness, blindness, hemiplegia) can make assessment difficult, as can cognitive impairment related to dementia, or narcotic analgesia administered in the prehospital phase.

Standardised analgesia protocols ensure that pain is properly assessed and appropriately treated, such that analgesia is provided without opioid-induced cognitive compromise. In turn, this facilitates other aspects of preoperative care, such as physical assessment, communication, eating and drinking and self-care.

Paracetamol (acetaminophen) is an effective analgesic that is well tolerated by hip fracture patients, and should be prescribed routinely throughout the perioperative period.

Renal dysfunction is common ( $40 \%)$ among this patient group, and so nonsteroidal anti-inflammatory drugs (and codeine and tramadol) should be used with caution, or avoided completely.

Opioid analgesics are effective, but can affect cognition and increasingly so with older age and/or declining renal function (in such patients the dose should be reduced and the dosing interval prolonged). Depending on the availability, buprenorphine, fentanyl and oxycodone may be preferable to morphine for long-term use. 
Preoperative peripheral nerve block has become generally accepted as an analgesic method that minimises the administration of cognition-impairing opioid analgesics [7]. The sensory innervation of the hip involves the femoral, obturator and sciatic nerves, and the skin surrounding the operative incision site, the lateral cutaneous nerve of the thigh. Femoral nerve block and fascia iliaca blocks have been used successfully to reduce pain and limit opioid use preoperatively. Although the efficacy of both blocks is improved by nerve stimulation and (more so by) ultrasound location [8], requiring additional equipment and expertise, both methods have proven to be relatively easy to learn by junior non-anaesthetists, and allied health professionals, such that their protocolised administration by orthogeriatricians should be possible without anaesthetic input. Although additional expertise is required, tunnelled femoral nerve/fascia iliaca catheters can be used to provide prolonged non-opioid analgesia in defined patients for whom surgery is not an option, or where surgery may be delayed for medical reasons.

\subsubsection{Preoperative Preparation}

Hip fracture patients are often frail and old, with multiple comorbidities demanding polypharmacy. Any of these factors alone or in combination may have contributed to the fall that preceded the fracture, but it is only rarely that the outcome benefits of attempting to improve any of these factors outweigh the risk of delaying surgery. Instead, anaesthetists need reassurance from orthogeriatricians that the patient is appropriately fit for anaesthesia and surgery-'normalised' rather than 'optimised'-and encouragement that risk is best managed by administering an appropriate anaesthetic. Orthogeriatricians should understand what an 'appropriate' anaesthetic involves (see later), and discuss this with anaesthetists who are less familiar with anaesthetising hip fracture patients, and so more likely to cancel patients for medical reasons, delaying surgery.

The Association of Anaesthetists guidelines detail common patient problems that can increase the risk of anaesthesia or its conduct, such as anticoagulation, valvular heart disease, pacemakers and electrolyte abnormalities, and recommend how these should be managed preoperatively [6]. Similarly, generic algorithms are available online that can be modified according to institutional protocols [9]. These are intended as aides-memoire for preoperative patient preparation, and are not intended to replace direct communication between anaesthetist and orthogeriatrician.

Most usefully, the Association guidelines identify acceptable and unacceptable reasons for delaying surgery in order to treat certain conditions (Table 8.1). Even so, 'acceptable' is not synonymous with 'obligatory', and surgery may still proceed even if these are present, if the additional risk is managed appropriately. These recommendations serve as a useful starting point when anaesthetists and orthogeriatricians convene to discuss the timing of surgery. 
Table 8.1 Acceptable and unacceptable reasons for delaying hip fracture surgery [6]

\begin{tabular}{l|l}
\hline Acceptable & Unacceptable \\
$\begin{array}{l}\text { - Haemoglobin concentration }<8 \mathrm{~g} \mathrm{dL}^{-1} \\
\text { - Plasma sodium concentration }<120 \mathrm{or}>150 \mathrm{mmol} \mathrm{L}^{-1} \text { and/or }\end{array}$ & $\begin{array}{l}\text { Lack of facilities or } \\
\text { theatre space }\end{array}$ \\
potassium concentration $<2.8$ or $>6.0 \mathrm{mmol} \mathrm{L}^{-1}$ & $\begin{array}{l}\text { Awaiting } \\
\text { echocardiography }\end{array}$ \\
- Uncontrolled diabetes & Unavailable surgical \\
- Uncontrolled or acute onset left ventricular failure & $\begin{array}{l}\text { Minor electrolyte } \\
\text { abnormalities }\end{array}$ \\
- Correctable cardiac arrhythmia with a ventricular rate $>120$ & \\
beats min $^{-1}$ & \\
- Chest infection with sepsis & \\
- Reversible coagulopathy &
\end{tabular}

Table 8.2 The Nottingham hip fracture score

\begin{tabular}{|c|c|c|c|}
\hline Variable & Points & $\begin{array}{l}\text { Total } \\
\text { score }\end{array}$ & $\begin{array}{l}\text { Predicted 30-day postoperative } \\
\text { mortality }(\%)\end{array}$ \\
\hline Age $66-85$ years & 3 & 0 & 0.4 \\
\hline Age 86 years or older & 4 & 1 & 0.6 \\
\hline Male & 1 & 2 & 1.0 \\
\hline $\begin{array}{l}\mathrm{Hb} \text { less than or equal to } 10 \mathrm{~g} \mathrm{dL}^{-1} \text { on } \\
\text { admission to hospital }\end{array}$ & 1 & 3 & 1.7 \\
\hline $\begin{array}{l}\text { Abbreviated mental test score } \leq 6 / 10 \text { at } \\
\text { hospital admission }\end{array}$ & 1 & 4 & 2.9 \\
\hline Living in an institution & 1 & 5 & 4.7 \\
\hline More than one co-morbidity* & 1 & 6 & 7.6 \\
\hline Active malignancy within last 20 years & 1 & 7 & 12.3 \\
\hline \multirow[t]{3}{*}{ Total score } & & 8 & 18.2 \\
\hline & & 9 & 27.0 \\
\hline & & 10 & 38.0 \\
\hline
\end{tabular}

A score out of ten is calculated by summating weighted points for eight criteria (left). The total score is used to predict the risk of a patient dying within 30 days of hip fracture surgery (right). Comorbidities (*) include myocardial infarction, angina, atrial fibrillation, valvular heart disease, hypertension, cerebrovascular accident, transient ischaemic attack, asthma, chronic obstructive pulmonary disease and renal dysfunction

\subsubsection{Ethical and Legal Considerations}

Hip fracture in elderly patients is associated with significant mortality, morbidity, psychosocial change and reduction in quality of life, although intraoperative mortality is uncommon $(<0.5 \%)$. Traditionally, discussion between doctors, patients and relatives about the risks and benefits of the various surgical options and recovery approaches has been limited, and hampered by difficulties quantifying risk. National validation of the Nottingham Hip Fracture Score (NHFS) (Table 8.2) supports its use as a risk adjustment for estimating 30-day mortality after hip fracture, in addition to other evidence for its value in predicting 1-year mortality and likelihood of early hospital discharge $[10,11]$. The NHFS serves as a useful starting point when discussing risk, but requires patient-specific adjustment. This is best achieved by preoperative communication between the anaesthetist and orthogeriatrician so that 
discussions with patients and their relatives accurately reflect the possible outcomes of their decisions about treatment.

Similarly, anaesthetists should be involved in discussions about perioperative resuscitation status and/or treatment boundaries, which should be confirmed before every patient undergoes surgery.

Anaesthetic input is also of value when developing patient information literature, for instance, describing what analgesia, antiemesis and anaesthesia interventions the patient can expect to receive.

\subsection{Intraoperative Care}

In a similar fashion to anaesthetists needing to understand the importance of frailty to orthogeriatric management, orthogeriatricians need to understand how anaesthesia affects postoperative outcome.

Anaesthesia delivered sympathetically to a patient's age, frailty and comorbidity can help re-enable patients after hip fracture surgery by improving analgesia, remobilisation, eating and drinking and cognitive function.

Ideally, in the immediate postoperative period, patients should be sitting up, conversing coherently, drinking and eating, pain free and disconnected from oxygen, intravenous fluids and urinary catheters (all of which impede remobilisation). Although it is not always possible to achieve each of these factors, the aim is to administer anaesthesia in such a way as to facilitate as many as possible.

Evidence for the effect of anaesthetic interventions remains limited. Previously, debate has centred mainly on whether general anaesthesia or spinal anaesthesia (with or without sedation) is preferable in terms of outcome. Randomised controlled trials have proved inconclusive for several reasons: 'general' and 'spinal' anaesthesia can describe a myriad of different techniques, a $2 \mathrm{~h}$ period of anaesthesia is probably unrelated to mortality 30 days later, early mortality (within) 5 days is an infrequent outcome for which very large trials would be needed to detect any difference, inclusion and exclusion criteria significantly affect selection bias, equipoise is lacking (most anaesthetists think one or other technique is 'best') and recruitment to follow up is complex [12]. Standardising the outcomes measured in studies should improve comparisons between techniques during meta-analyses [13]. With the advent of 'Big Data', regional and national observational studies have been conducted, but have so far failed to find consistent benefits of one technique over another, at least in terms of mortality $[1,2,14]$.

\subsubsection{General or Spinal Anaesthesia?}

General anaesthesia involves the administration of narcotic and hypnotic anaesthetic agents that render a patient unconscious for the duration of surgery. The patient requires airway support, regardless of whether they are allowed to breathe spontaneously or are paralysed and their lungs ventilated artificially. 
Spinal anaesthesia is effectively a reverse dural tap, in which $1-3 \mathrm{~mL}$ of local anaesthetic (usually bupivacaine) is injected through a fine bore needle into the subarachnoid cerebrospinal fluid in the lumbar region, providing analgesia, akinesia and anaesthesia below the umbilicus for several hours. Additional sedation is usually administered, either as a bolus or continuously.

Recent meta-analyses, RCTs and large observational studies report conflicting results about whether mortality is lower after general or spinal anaesthesia $[2,3$, 14]. However, there is greater consensus in terms of postoperative morbidity and cost, favouring spinal over general anaesthesia. Anecdotally, anaesthetists would prefer to have spinal anaesthesia themselves if they needed hip fracture surgery, orthogeriatricians report better patient recovery after spinal anaesthesia, and physiotherapists report easier patient remobilisation after spinal anaesthesia. Results reported from imminent RCTs (REGAIN, REGARD, iHOPE) should add further information to this determination.

However, of greater relevance than whether spinal or general anaesthesia is better for patients is how well that anaesthesia is delivered. Although there are theoretical and experimental reasons for avoiding general anaesthesia (and sedation) in the elderly, the effect of these is seemingly small compared to numerous other adverse effects of anaesthesia and surgery, including hypotension, pain and analgesia, hypoxia and anaemia. Instead, anaesthetists should focus on careful monitoring of patients during surgery and the provision of appropriate interventions to normalise physiology, for example fluid and vasopressor therapy, depth of anaesthesia/cerebral oxygenation monitoring.

Future research has begun to focus on early postoperative outcomes that are more anaesthesia-specific, such as pain, hypotension and delirium (e.g., ASCRIBED, HIP-HOP and RAGA-delirium RCTs), and clearer definition of the anaesthetic techniques compared (e.g., self-ventilating general anaesthesia + nerve block vs. opioid-free, low-dose spinal anaesthesia + local anaesthetic infiltration without sedation).

\subsubsection{Peripheral Nerve Block}

Peripheral nerve blockade (fascia iliaca, femoral nerve, lumbar plexus blocks, or local anaesthesia infiltration) should always be administered with either general or spinal anaesthesia, as part of a multimodal analgesia protocol that aims to minimise opioid co-administration $[5,6,15,16]$.

Theoretically, fascia iliaca blocks may provide better analgesia of both the hip and surgical incision site intraoperatively, without dense blockade of the femoral nerve, which can prolong and impair remobilisation. Administered beforehand, a fascia iliaca or femoral nerve block can reduce sedation requirements when positioning patients laterally for spinal anaesthesia administration, and precludes the need to co-administer subarachnoid opioids, which can cause itching, respiratory depression and urinary retention postoperatively.

Co-administration of peripheral nerve blockade beforehand reduces age-adjusted maintenance doses of general anaesthesia. 


\subsubsection{Spinal Anaesthesia}

The aim of spinal anaesthesia is to achieve unilateral blockade on the operative side to a sensory level of $\sim T_{10-12}$ for $\sim 2 \mathrm{~h}$ maximum operating time, whilst avoiding excessive hypotension related to spinal-induced sympatholysis. This can be achieved using opioid-free 1-1.5 mL subarachnoid 0.5\% hyperbaric bupivacaine [17], but these doses are administered to fewer than $20 \%$ of patients receiving spinal anaesthesia. Instead, anaesthetists commonly administer in excess of $2 \mathrm{~mL} 0.5 \%$ bupivacaine $[14,18]$, which is associated with greater relative falls in blood pressure from pre-spinal baseline and a wider range of blood pressure reductions compared to lower doses, changes that can persist into the early postoperative period and prevent patients from sitting out of bed or standing up after surgery. Orthogeriatricians have an important role in encouraging anaesthetists at their institutions to use lower doses of spinal anaesthesia.

\subsubsection{Sedation}

Similarly, orthogeriatricians have a role in encouraging anaesthetists to consider using less, or no, sedation during spinal anaesthesia.

Commonly, patients co-administered spinal anaesthesia and peripheral nerve block sleep through surgery, because the relative narcotic effect of preoperative opioids increase when pain is alleviated during spinal anaesthesia, and patients are often sleep deprived from the night preceding surgery.

If patients request sedation or sedation is necessary for patient comfort and immobility during surgery, then the minimum amount should be used for the shortest time, to avoid accumulation and sedation in the postoperative period.

Several papers have shown that sedative infusions result in general anaesthesia (without airway support) in a significant proportion of hip fracture patients [19], and so sedation may better be limited to small bolus administration during key periods of surgery (jigsawing, hammering, relocation). Depth of anaesthesia monitors should probably be used to guide sedation if infusions are to be administered.

Theoretically, propofol is the sedative of choice, as it is metabolised rapidly, its metabolites are inert (unlike midazolam) and it does not cause prolonged cognitive impairment (unlike ketamine). There is no evidence supporting the use of combinations of sedatives, although this is common practice.

\subsubsection{General Anaesthesia}

Older patients are sensitive to the cardiovascular effects of general anaesthesia (negative inochronotropicity and peripheral vasodilation). Hypotension is more common during general anaesthesia compared to spinal anaesthesia, but decreasing the amount of inhalational or intravenous anaesthetic agent administered during surgery can reduce its prevalence. Moreover, compared to younger patients, the elderly 
require lower doses of drugs to maintain anaesthesia, particularly when a peripheral nerve block is administered preoperatively.

Minimising hypotension while maintaining anaesthesia without awareness can be achieved using depth of anaesthesia monitors (e.g., bispectral index (BIS) and E-Entropy), and it has been recommended that these be used during any type of general anaesthesia in older patients [5, 6]. Alternatively, a Lerou nomogram can be used to adjust inhalational anaesthesia agent dose for age, or age-adjusted doses programmed into a total intravenous anaesthesia syringe pump.

One of the enduring debates among anaesthetists concerns whether the airway of a hip fracture patient administered general anaesthesia should be supported using a laryngeal mask airway, thereby avoiding the pathophysiological effects of mechanical ventilation, or should be intubated, to avoid the risk and consequences of aspiration pneumonia. Respiratory failure is significantly more prevalent after general compared to spinal anaesthesia, and use of paralysing agents is dose-dependently associated within an increased risk of postoperative respiratory complications, but it remains unclear whether hip fracture patients benefit more by avoiding aspiration or by avoiding mechanical ventilation.

\subsubsection{Avoiding Ischaemia}

Both general and spinal anaesthesia are associated with a high prevalence of hypotension during anaesthesia for hip fracture surgery, general more so than spinal anaesthesia, and postoperative mortality correlates with increased relative fall in blood pressure [14, 18]. Hypotension can be predicted, and ameliorated by administering less anaesthesia, monitoring blood pressure closely, avoiding preoperative dehydration, and administering fluids and vasopressors appropriately.

Hypothetically, avoiding hypotension should reduce the prevalence of postoperative complications related to organ ischaemia, such as confusion/delirium [20], dysrhythmia, acute kidney injury and poor remobilisation. Ischaemic complications may further be attenuated by ensuring adequate postoperative oxygen saturations (e.g., by providing (nasal) oxygen if $\mathrm{SpO}_{2} \leq 95 \%$ ), avoiding excessive anaemia (e.g., by measuring blood haemoglobin concentration immediately after surgery and on day 1 , and considering transfusion) and providing adequate pain relief (to reduce oxygen uptake). Note that simply by reducing anaesthetic dose reduces the prevalence of hypotension, requiring reduced fluid administration, in turn causing less dilutional anaemia, and so, in combination with additional peripheral nerve blockade, less ischaemia.

\subsubsection{Bone Cement Implantation Syndrome (BCIS)}

BCIS describes a complication occurring during surgical instrumentation and/or cementing of the femoral canal, and is characterised by cardiorespiratory compromise/arrest. It occurs in about $20 \%$ of hip fracture operations in which cement is used, and results in cardiopulmonary arrest in about $0.5 \%[19,21]$. 
The AAGBI, British Geriatric Society and British Orthopaedic Association have published multidisciplinary guidelines highlighting the need for joint decisionmaking, team working and attention to detail during the peri-operative period [22].

Of particular importance is the need to identify patients who are at higher risk of BCIS, including those who are very elderly, male, taking diuretic medication and have comorbid cardiorespiratory disease (particularly acute lung pathology).

Compared to uncemented prostheses, the use of cemented prostheses for hip fracture repair increases the likelihood of pain-free mobility after surgery and reduces the risk of re-operation. However, the guidelines recommend that surgeons, anaesthetists and orthogeriatricians discuss preoperatively whether the benefits of using a cemented prosthesis outweigh the risk of BCIS.

\subsubsection{Standardisation of Anaesthesia}

Clinical outcomes and other measures of care quality have gradually improved in the United Kingdom after hip fracture repair over the last decade. This has resulted from the general standardisation of care, with payments to hospitals for care supplemented by a bonus if they are sure that the defined care targets were met ('payment by results'). Conspicuously absent are targets related to anaesthesia, which combined with an ongoing lack of research evidence and lack of formal professional training in how to anaesthetise hip fracture patients, has meant that there continues to be a wide national variation in anaesthesia practice for hip fracture $[1,2,12,15]$.

Of course, a lack of standardisation may not matter-anaesthesia may have little effect on outcome after hip fracture-but this is unlikely to be the case, given that anaesthesia is administered at the most critical phase of a patient's recovery after hip fracture and has an immediate effect on the trajectory of recovery postoperatively. Until better evidence becomes available, it seems prudent, however, to reject the tacit acceptance of poor, outlying care in support of current evidence-based standardised care as a method for improving safety, in a similar fashion to providing standardised anaesthesia care as part of Enhanced Recovery Protocols.

Although there is some evidence supporting the use of protocolised rather than physician-individualised, there is no evidence supporting physician-individualised care over protocolised care.

In healthcare, standardisation is particularly beneficial when implementing evidence-based care for large numbers of patients with a similar disease process, for whom current treatment is costly, has poor outcomes and is recognised professionally as being of sub-optimal quality - all of which apply in hip fracture.

Standardisation ensures high reliability, consistent, cheaper, higher quality care for the majority of patients, and — most importantly - that the basics of care are not overlooked. Furthermore, standardisation enables monitoring and continuous improvement by amending standards in an evidence-based fashion, reductions in artificial variations in care (caused by slips, lapses or lack of knowledge) whilst improving focus on natural variation in care (caused by differences between 
patients) and identification of consistently poor performance, areas for future research and educational needs.

Standards for anaesthesia are currently available online (www.hipfractureanaesthesia.com), based on best available current evidence and consensus opinion, describing the rationale behind their formulation and identifying areas for further research. As developed, these standards also provide a method of understanding why individual anaesthetists have deviated from standard practice.

Orthogeriatricians are encouraged to engage anaesthetic colleagues in following these standards, undertaking research in improving them further and engaging in continuous quality improvement cycles, with the aim of optimising care in the critical early postoperative period. This is a mutually cooperative process, as anaesthetists and orthogeriatricians should work together to measure and monitor preoperative care, with the aim of optimising the patient pathway from fracture to early surgical fixation.

\subsection{Postoperative Care}

Much of anaesthetic involvement in the postoperative phase has been described earlier. Irrespective of whether the patient has been administered general or spinal anaesthetic (with/without sedation), the orthogeriatrician should expect to receive a patient back on the acute orthopaedic ward/hip fracture unit who is immediately ready for re-enablement (resuming activities of daily living) and suitable for rehabilitation to their former place of residence.

The 2012/20 AAGBI guidelines detail the management of common early postoperative complications, including pain, oxygenation, fluid balance and delirium [6]. These are essentially continuations of the primary aims of anaesthesia in the hip fracture population, namely the avoidance of 'ischaemia' through appropriate pain, blood pressure, oxygen, fluid and blood management, so that the consequences of 'ischaemia' - delirium [23], heart pump or rhythm disturbance, acute kidney injury, delay in remobilisation-are avoided.

Gut disturbances are common after hip fracture surgery and often overlooked. Nausea and vomiting delay resumption of oral feeding. Constipation occurs in the majority of patients, particularly those who are dehydrated, not eating or dehydrated. Malnutrition is common especially in frail patients and the cognitively impaired, and close attention to dietary intake is essential to patients' re-enablement.

The role of high dependency or intensive care remains uncertain after hip fracture. Certainly, it is never ethically justified to deny access to these facilities based on a hip fracture patient's age, and in any other group of patients with a similar 30-day postoperative mortality (or indeed mortality $>1 \%$, e.g., patients requiring emergency laparotomy), critical care facilities are much more routinely accessed. Indeed, planned admission is important in patients with a pre-operatively identifiable need for single/dual system support postoperatively, when this cannot be 
achieved to the same degree on an acute orthopaedic ward; for example patients with COPD, acute lung injuries (infection, embolism) and acute left ventricular failure will benefit from critical care. Patients for whom critical care admission is planned have good outcomes compared to patients for whom critical care admission is unplanned, but this reflects the likelihood of intraoperative complications such as bone cement implantation syndrome, on table cardiac arrest or cerebrovascular accident, or massive haemorrhage.

However, adopting systems of orthogeriatric care allows a greater number of elderly patients with comorbidities to receive 'acute' medical care on acute orthopaedic wards after hip fracture surgery, rather than taxing precious critical care resources. Furthermore, orthogeriatric services are able to coordinate step-down care, reducing the duration of critical care admission. Having managed the patient preoperatively, orthogeriatricians may have a more pragmatic approach to normalising patients back to their previous physiological condition, in comparison to the more critical care approach of optimising organ function, although this assertion requires further research.

\section{References}

1. National Hip Fracture Database (2019) Annual Report 2018. http://www.nhfd.co.uk/ files/2018ReportFiles/NHFD-2018-Annual-Report-v101.pdf. Accessed 1 Oct 2019

2. White SM, Moppett IK, Griffiths R (2014) Outcome by mode of anaesthesia for hip fracture surgery. An observational audit of 65535 patients in a national dataset. Anaesthesia 69:224-230

3. Parker M, Handoll HHG, Griffiths R (2016) Anaesthesia for hip fracture surgery in adults. Cochrane Database Syst Rev 4:CD000521

4. Luger TJ, Kammerlander C, Gosch M et al (2010) Neuroaxial versus general anaesthesia in geriatric patients for hip fracture surgery: does it matter? Osteoporos Int 21:S555-S572

5. White SM, Altermatt F, Barry J et al (2018) International Fragility Fracture Network consensus statement on the principles of anaesthesia for patients with hip fracture. Anaesthesia 73:863-874

6. Association of Anaesthetists (2020) Management of hip fractures 2020. Currently under review

7. Guay J, Parker MJ, Griffiths R, Kopp SL (2017) Peripheral nerve blocks for hip fractures: a Cochrane review. Cochrane Database Syst Rev 1:CD001159

8. Dolan J, Williams A, Murney E, Smith M, Kenny GN (2008) Ultrasound guided fascia iliaca block: a comparison with the loss of resistance technique. Reg Anesth Pain Med 33:526-531

9. White SM (2019) Hip fracture anaesthesia. www.hipfracture.anaesthesia.co.uk. Accessed 25 Oct 2019

10. Moppett IK, Parker M, Griffiths R, Bowers T, White SM, Moran CG (2012) Nottingham Hip Fracture Score: longitudinal and multi-centre assessment. Br J Anaesth 109:546-550

11. Marufu TC, White SM, Griffiths R, Moonesinghe SR, Moppett IK (2016) Prediction of 30-day mortality after hip fracture surgery by the Nottingham hip fracture score and the surgical outcome risk tool. Anaesthesia 71:515-521

12. White SM, Griffiths R, Moppett I (2012) Type of anaesthesia for hip fracture surgery-the problems of trial design. Anaesthesia 67:574-578

13. O'Donnell CM, Black N, McCourt KC et al (2019) Development of a Core Outcome Set for studies evaluating the effects of anaesthesia on perioperative morbidity and mortality following hip fracture surgery. Br J Anaesth 122:120-130 
14. White SM, Moppett IK, Griffiths R et al (2016) Secondary analysis of outcomes after 11,085 hip fracture operations from the prospective UK Anaesthesia Sprint Audit of Practice (ASAP-2). Anaesthesia 71:506-514

15. The National Institute of Clinical Excellence. Clinical Guideline 124 (2011) The management of hip fracture in adults. http://www.nice.org.uk/nicemedia/live/13489/54918/54918.pdf. Accessed 1 Apr 2016

16. Scottish Intercollegiate Guidelines Network (2009) Management of hip fracture in older people. National clinical guideline 111. www.sign.ac.uk/pdf/sign111.pdf. Accessed 1 Apr 2016

17. Wood RJ, White SM (2011) Anaesthesia for 1131 patients undergoing proximal femoral fracture repair: a retrospective, observational study of effects on blood pressure, fluid administration and perioperative anaemia. Anaesthesia 66:1017-1022

18. Royal College of Physicians and the Association of Anaesthetists of Great Britain and Ireland (2014) National Hip Fracture Database. Anaesthesia Sprint Audit of Practice 2014. http:// www.nhfd.co.uk/20/hipfractureR.nsf/4e9601565a8ebbaa802579ea0035b25d/f085c66488 1d370c80257cac00266845/\$FILE/onlineASAP.pdf. Accessed 1 Apr 2016

19. Sieber FE, Gottshalk A, akriya KJ, Mears SC, Lee H (2010) General anesthesia occurs frequently in elderly patients during propofol-based sedation and spinal anesthesia. J Clin Anesth 22:179-183

20. Ballard C, Jones E, Gauge N et al (2012) Optimised anaesthesia to reduce post operative cognitive decline (POCD) in older patients undergoing elective surgery, a randomised controlled trial. PLoS One 7:e37410

21. Donaldson AJ, Thomson HE, Harper NJ, Kenny NW (2009) Bone cement implantation syndrome. Br J Anaesth 102:12-22

22. Griffiths R, White SM, Moppett IK et al (2015) Association of Anaesthetists of Great Britain and Ireland; British Orthopaedic Association; British Geriatric Society. Safety guideline: reducing the risk from cemented hemiarthroplasty for hip fracture 2015: Association of Anaesthetists of Great Britain and Ireland British Orthopaedic Association British Geriatric Society. Anaesthesia 70:623-626

23. Chuan A, Zhao L, Tillekeratne N, Alani S, Middleton PM, Harris IA, McEvoy L, Ní Chróinín D (2019) The effect of a multidisciplinary care bundle on the incidence of delirium after hip fracture surgery: a quality improvement study. Anaesthesia. https://doi.org/10.1111/anae.14840

Open Access This book is licensed under the terms of the Creative Commons AttributionNonCommercial-NoDerivatives 4.0 International License (http://creativecommons.org/licenses/ by-nc-nd/4.0/), which permits any noncommercial use, sharing, distribution and reproduction in any medium or format, as long as you give appropriate credit to the original author(s) and the source, provide a link to the Creative Commons license and indicate if you modified the licensed material. You do not have permission under this license to share adapted material derived from this book or parts of it.

The images or other third party material in this book are included in the book's Creative Commons license, unless indicated otherwise in a credit line to the material. If material is not included in the book's Creative Commons license and your intended use is not permitted by statutory regulation or exceeds the permitted use, you will need to obtain permission directly from the copyright holder.

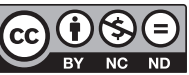

